\title{
Optimal Sensor Placement for Damage Detection: Role of Global Search
}

\author{
Prakash Kripakaran, Sandro Saitta, Suraj Ravindran, Ian F. C. Smith \\ Structural Engineering Institute, Ecole Polytechnique Fédérale de Lausanne, Switzerland \\ E-mail : prakash.kripakaran@epfl.ch
}

\begin{abstract}
Optimal sensor placement is one that maximizes the likelihood of identifying future damage models. Based on assumptions from engineers, damage models of a structure are simulated and their predictions are computed. Computational approaches are used to place sensors at locations that maximize the chances of identifying damage. This paper studies the application of global search for optimal sensor placement. The global search methodology uses stochastic sampling to find optimal locations for sensors. In a previous study, Robert-Nicoud et al. proposed a greedy strategy that places sensors sequentially at locations where model predictions have maximum entropy. Performance of the two strategies are compared for the Schwandbach bridge in Switzerland. The results show that global search is better for designing measurement systems on a previously unmonitored structure while the greedy algorithm is better for incremental measurementinterpretation strategies.
\end{abstract}

\section{Introduction}

There are hundreds of ways to measure physical phenomena in structures and many new measurement technologies are emerging every year. However, inferring meaningful information from data remains a major difficulty. A systematic approach to interpretation of measurement data employs methodologies developed in the field of system identification [1]. System identification involves determining the state of a system and values of system parameters through comparisons of predictions with measurements. Once identified, models can support decision-making with respect to future maintenance and repair. Since system identification is an inverse problem and errors are involved in both measurement and modeling, many models may be able to explain the same measurement [2]. Therefore it is of interest to configure measurement systems such that maximum separation between candidate models can be achieved [3].

Sensors are increasingly used for tasks such as fault diagnosis [4] and automatic control [5]. Civil engineering examples are bridge diagnosis [6] and construction quality control [7]. The configuration of sensors within these systems remains a task that engineers perform often without systematic and scientific evaluation. The field of sensor configuration is at an early research stage and the subject of sensor networks is now emerging in parallel [5]. Currently, engineers often make ad hoc decisions related to location and types of sensors to be used.

The main objective of employing sensors on infrastructure systems such as bridges is damage detection. For successful damage detection, it is essential that appropriate sensors are placed at locations such that the chances of detecting damage are maximized. Damage scenarios to be considered depend on factors such as the material, the structural system, boundary conditions, loads and geographical location. For instance, one of the piers in a bridge may not be functioning as a support after a flood or an earthquake. Combinations of different scenarios are also possible. Optimal sensor placement is one that gives maximum separation between predictions of the effects of damage scenarios.

In this paper, configuring a measurement system is considered to be a discrete combinatorial optimization problem. Indeed, the number of sensors to be used as well as the number of possible sensor locations are discrete variables. The complexity of the solution is combinatorial since each sensor can be placed anywhere although only once. Robert-Nicoud et al. [8] suggested a greedy strategy for sensor placement that uses the entropy among model predictions to identify optimal sensor locations. In greedy algorithms, strategies that accept a less attractive local alternative for a better overall solution do not exist. Although finding the globally optimal solution for some optimization problems, greedy algorithms may find non optimal solutions for other problems. 
Unlike greedy algorithms, global search algorithms [9-11] aim to find the best solution among all possible. This paper explores the role of global search in measurement system design using the Schwandbach bridge in Switzerland as a representative structure. Section 2 formally defines the problem of optimal sensor placement. The greedy algorithm and global search approach to find the best sensor locations are described. Section 3 describes the Schwandbach bridge that is used to illustrate the sensor placement algorithms. Section 4 discusses results from both greedy algorithm and global search. Section 5 lists the conclusions from the study.

\section{Optimal Sensor Placement}

Sensor-data driven decision support systems can be the basis of proactive management of structural facilities [12]. For effective decision support, it is essential that sensor systems are configured to measure responses at locations such that the measured data can be meaningfully interpreted.

Robert-Nicoud et al. [13] developed a sensor placement methodology that consists of two key steps (1) Generation of candidate models and (2) Sensor performance evaluation at each location. Since generating all possible models is combinatorial, a population of models is randomly generated using assumptions declared by the engineer. Each model is evaluated by finite element analysis. Its predictions $p_{i}$ at all possible sensor locations are computed and stored in a set $M_{0}$. The number $N$ of sampled models depends upon the modeling assumptions and engineer preferences. Thus, there are $N$ sets of predictions $p$ in $M_{0}$.

The goal of measurement system configuration is to place sensors at locations that offer maximum separability between these $N$ model predictions. Given sensor configuration with $s$ number of sensors, its performance is evaluated as follows. Depending upon the precision of the sensor and the model predictions in $M_{0}$, a suitable number of intervals $I$ is identified for classifying predictions at each potential sensor location. At each location $i$ where a sensor is placed, a histogram with $I$ intervals is built for the model predictions in $M_{0}$. Each bar in the histogram represents the number of models whose predictions lie within the corresponding interval. Let $B_{i}$ represent a set of subsets where each subset contains predictions in a bar of histogram at location $i$. Thus, $B_{1}, B_{2} \ldots B_{s}$ represent the corresponding sets obtained by evaluating histograms at sensor locations 1 to $s$. Then the maximum number of non-identifiable models $U_{\max }$ is given as the maximum possible size of the set $\mathrm{B}$ given by

$$
B=\left\{b_{1} \cap b_{2} \cap b_{3} \ldots \cap b_{s}\right\}
$$

$b_{i}$ represents an element of set $B_{i}$. Thus the objective of the optimal sensor placement problem is to minimize the value of $U_{\max }$. The following sections describe two strategies to identify sensor locations that minimize $U_{\max }$.

\subsection{Greedy Algorithm}

The greedy algorithm places a sensor at the location that best separates the biggest subset of model predictions. It does not allow for subsequent relocation when more sensors are added. To measure the separation between models, [8] uses the notion of entropy. Shannon's entropy function [14] is a mathematical representation for the uncertainty in a set. This expression comes from the field of information theory and it formulates the disorder within a set. In our case, a set is an ensemble of model predictions for a system identification task. For a random variable $\mathrm{X}$, the entropy $\mathrm{H}(\mathrm{X})$ is given by the following equation.

$$
H(X)=-\sum_{i=1}^{|X|} P_{i} \cdot \log \left(P_{i}\right)
$$

$P_{i}$ are the probabilities of the $|X|$ different possible values of $X$. For practical purposes, $0 \cdot \log (0)$ is taken to be 0 . The maximum of the entropy function is at $\mathrm{P}=$ 0.5 when the probability is equally distributed between the two values. When a variable takes $|X|$ discrete values, the entropy is a maximum when all values have the same probability $\log _{2}(X)$. Thus entropy is a measure of homogeneity in a distribution. A completely homogenous distribution has maximum entropy. The entropy for a given sensor location is calculated for the histogram of model predictions. The probability $P_{i}$ of an interval is the ratio of the number of models in the interval to the total number of models. At the best measurement locations, model predictions should have maximum variation.

The greedy algorithm for sensor placement is described in the flowchart given in Figure 1. The algorithm iteratively chooses locations with highest entropy for sensor placement. The algorithm stops when there is no further improvement to the maximum number of non-identifiable models, i.e., $M_{\max }$ does not become smaller. 


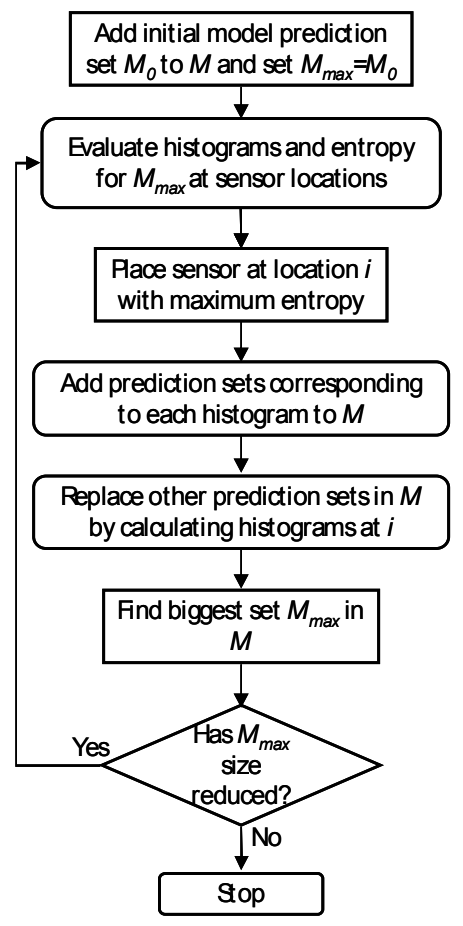

\section{Figure 1: Flowchart of greedy algorithm for sensor placement}

\subsection{Analysis of Complexity}

In the greedy algorithm, previously selected sensor locations are not permitted to change when the next best sensor is determined. This is the principal drawback of the greedy solution. Strategies that accept a less attractive intermediate solution for a better overall solution are not allowed. A more rigorous approach is to test all possible configurations for sensor placement. If $l$ sensors are to be placed on the system, all combinations of $l$ sensors among $L$ possible locations are tested. Then the computational complexity of the task to determine the optimal number of sensors and their locations is given by

$$
\sum_{l=1}^{L} C_{L}^{l}=2^{L}-1
$$

The task of placing $l$ sensors among $L$ possible locations is combinatorial and the total complexity is exponential as shown by Equation (2). It is obvious that trying all possible solutions for the measurement system gives the best configuration. However, as explained in [7] the number of possible sensor locations can be extremely high. This makes the calculation of all possible configurations infeasible. Therefore, a global search algorithm is used. PGSL [16] is chosen due to its efficiency and ease of use.

\subsection{Global Search (PGSL)}

PGSL [11, 15] is a direct search algorithm that employs global sampling to find the minimum of a user defined objective function. Gradient calculations are not needed and no special characteristics of the objective functions (such as convexity) are required. PGSL has been successfully applied to optimization problems in structural control [16] and system identification [13].

Primary input to PGSL is the number of variables and the range of acceptable values for each variable. For the sensor placement problem, the number of decision variables is equal to the number of potential sensor locations. The stochastic sampling nature of PGSL means that it operates only on continuous variables. However, the variables for the sensor placement problem are binary decision variables representing the presence or absence of a sensor at each sensor location. To overcome this problem, each variable is modeled as continuous and varying between 0 and 1 in PGSL. Consider the case when PGSL is used to find the optimal sensor locations for number of sensors equal to $I$. Then each solution generated by PGSL is interpreted as having sensors only at those locations corresponding to variables with the $I$ largest values.

\section{Representative Bridge}

A bridge that was designed by Maillart in 1933 (Figure 2) is used to illustrate the sensor configuration methodology. Still standing today, the Schwandbach Bridge is an early example of a deck-stiffened openspandrel arch and has been named by [17] "to be one of the two or three most beautiful concrete bridges ever built".

A set of possible sensor locations on the bridge are shown in Figure 3 using node numbers. Since this is a bridge with a short span of approximately $50 \mathrm{~m}$, the deflections of the bridge are relatively small. Displacement sensors that can measure with an accuracy of up to $1 \mathrm{~mm}$ are found to be insufficient for system identification. However, inclinometers with fairly high accuracy (approximately $1 \mu R$ ) are available and this resolution is observed to be acceptable for performing system identification. In the following section, damage scenarios that determine the location of the inclinometers on the bridge are explained. 


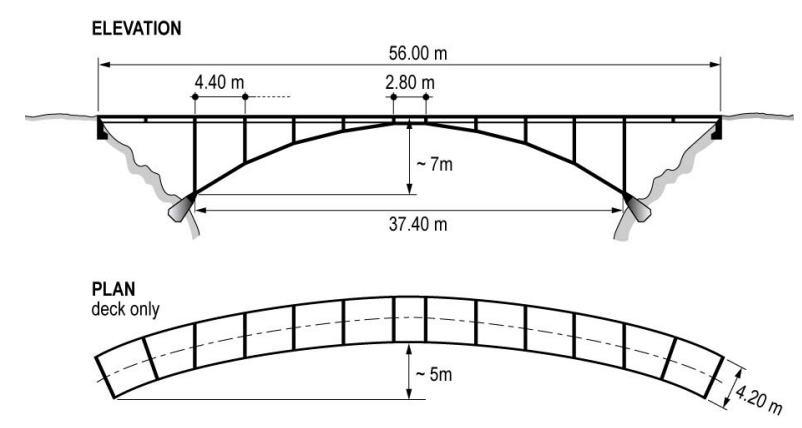

Figure 2: Schema of the Schwandbach bridge

The damage scenarios that are used in this case study are listed in Table 1 . The scenarios are derived from a previous experimental study on the Z24 bridge in Switzerland by Maeck et al. [18]. Table 1 also lists the degree of damage to be simulated under each scenario. In this study, the bridge is modeled as a finite element model in ANSYS. Damage scenarios are simulated by appropriately changing the parameters of the finite element model. Candidate models are generated by stochastic sampling in a model space that consists of all combinations of the damage scenarios listed in Table 1.

\section{Results}

A set containing 5000 damage models of Schwandbach Bridge is created in order to represent the space of possible models. The number of possible sensor locations is 20 (see Figure 2 for details). The size of the solution space is $2^{20}$ since a sensor may or may not be present at a given location. This space is sufficiently large to illustrate key aspects of the methodology.

Schwandbach Bridge is nearly symmetrical with respect to a center line (depicted by line $\mathrm{X}-\mathrm{X}$ in Figure 2) and the damage scenarios considered in this study are also symmetrical. Therefore, the sensor placement algorithms explicitly impose symmetry by placing a pair of sensors in each iteration.

Both greedy strategy and global search are used for sensor placement. The number of intervals $I$ depends on the sensor precision. For this simulation, $\mathrm{I}=10$. Table 2 shows the results obtained from the two strategies. For every consecutive set of sensors placed, the size of the biggest subset of non identifiable (nonid.) models is given in Table 2. The global search is able to generate better sensor configurations than the greedy strategy when the number of sensors is greater than 1. Also while global search is able to achieve maximum identifiability with only 10 sensors, greedy

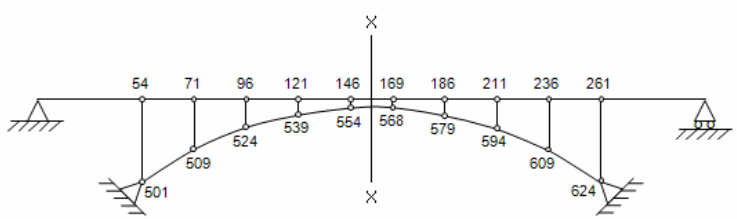

\section{Figure 3: Potential sensor locations on Schwandbach bridge}

strategy proposes a configuration requiring 14 sensors. Further reduction in the number of non-identifiable models would require use of new sensor types or inclusion of new sensor locations in the sensor placement strategy.

Table 1: Damage scenarios considered

\begin{tabular}{l|l}
\hline Damage & Damage limits \\
\hline Arch abutment settlement & $0-30 \mathrm{~cm}$ \\
Arch abutment tilt & $0-2^{\circ}$ \\
Deck support settlement & $0-30 \mathrm{~cm}$ \\
Cracks on inner girder & $0-50 \%$ reduction \\
Spalling on inner girder & $0-50 \%$ reduction \\
Spalling on outer girder & $0-50 \%$ reduction \\
\hline
\end{tabular}

Table 2: Comparison of greedy and global strategies

\begin{tabular}{l|c|c}
\hline \multirow{2}{*}{$\begin{array}{l}\text { Number } \\
\text { of } \\
\text { sensors }\end{array}$} & \multicolumn{2}{|c}{ Non-id. models \# } \\
\cline { 2 - 3 } & $\begin{array}{l}\text { Greedy } \\
\text { strategy }\end{array}$ & $\begin{array}{c}\text { Global } \\
\text { search }\end{array}$ \\
\hline 2 & 181 & 181 \\
4 & 113 & 111 \\
6 & 86 & 76 \\
8 & 80 & 58 \\
10 & 63 & 51 \\
12 & 54 & 51 \\
14 & 51 & 51 \\
16 & 51 & 51 \\
\hline
\end{tabular}

In spite of being less attractive for initial sensor configuration, the greedy strategy is the only option when making decisions related to additional measurements on structures that already contain sensors. Since civil engineering structures are often designed to last over a hundred years, incremental measurement-interpretation strategies are expected to be normal practice. Therefore, both configuration strategies are useful. 


\section{Conclusions}

The conclusions of this research are

- Global search is a better option for sensor placement than a greedy strategy when configuring new measurement systems for structures

- A greedy strategy is useful when augmenting an existing measurement system with additional sensors

Future research in measurement system design involves extending sensor placement algorithms to accommodate multiple sensor types. The inclusion of model predictions corresponding to variations in modeling assumptions in the initial model set is being investigated. The effect of measurement errors and sensor types with different precisions is also current research.

\section{Acknowledgments}

This work is funded by the Swiss National Science Foundation under contract no. 200020-109257. The authors would like to thank B. Raphael for discussions.

\section{References}

[1] L. Ljung, System Identification - Theory for the User: Prentice Hall, 1999.

[2] I. F. C. Smith, "Sensors, Models and Videotape," presented at Computing in Civil Engineering 2005, Cancun, Mexico, 2005.

[3] S. Saitta, B. Raphael, and I. F. C. Smith, "Rational design of measurement systems using information science," presented at IABSE Conference, Budapest, 2006.

[4] J. A. Camelio, S. J. Hu, and H. Yim, "Sensor Placement for Effective Diagnosis of Multiple Faults in Fixturing of Compliant Parts," Journal of Manufacturing Science and Engineering, vol. 127, pp. 68-74, 2005.

[5] D. E. Culler and W. Hong, "Wireless Sensor Networks," Communications of the ACM, vol. 47, pp. 32-33, 2004

[6] Y. Robert-Nicoud, B. Raphael, O. Burdet, and I. F. C. Smith, "Model Identification of Bridges Using Measurement Data," Computer-Aided Civil and Infrastructure Engineering, vol. 20, pp. 118-131, 2005.

[7] B. Akinci, F. Boukamp, C. Gordon, D. Huber, C. Lyons, and K. Park, "A formalism for utilization of sensor systems and integrated project models for active construction quality control," Automation in Construction, vol. 15, pp. 124-138, 2006.

[8] Y. Robert-Nicoud, B. Raphael, and I. F. C. Smith, "Configuration of measurement systems using Shannon's entropy function," Computers \& Structures, vol. 83, pp. 599-612, 2005.

[9] S. Kirkpatrick, C. D. Gelatt, Jr., and M. P. Vecchi, "Optimization by Simulated Annealing," Science, vol. 220, pp. 671-680, 1983.

[10] D. E. Goldberg, Genetic Algorithms in Search, Optimization and Machine Learning: Addison-Wesley Professional, 1989.

[11] B. Raphael and I. F. C. Smith, "Engineering applications of a direct search algorithm, PGSL," presented at 2005 ASCE Computing Conference, Reston VA, USA, 2005.

[12] J. H. Garrett, B. Akinci, S. Matthews, C. Gordon, H. J. Wang, and V. Singhvi, "Sensor data driven proactive management of infrastructure systems," in Intelligent Computing in Engineering and Architecture, vol. 4200, Lecture Notes in Artificial Intelligence, 2006, pp. 262-284.

[13] Y. Robert-Nicoud, B. Raphael, and I. F. C. Smith, "System Identification through Model Composition and Stochastic Search," Journal of Computing in Civil Engineering, vol. 19, pp. 239-247, 2005.

[14] C. Shannon and W. Weaver, The Mathematical Theory of Communication: University of Illinois Press, 1949.

[15] B. Raphael and I. F. C. Smith, "A direct stochastic algorithm for global search," Applied Mathematics and Computation, vol. 146, pp. 729-758, 2003.

[16] B. Domer, B. Raphael, K. Shea, and I. F. C. Smith, "A Study of Two Stochastic Search Methods for Structural Control," Journal of Computing in Civil Engineering, vol. 17, pp. 132-141, 2003.

[17] D. Billington, Robert Maillart's Bridges: Princeton University Press, 1979.

[18] J. Maeck, B. Peeters, and G. De Roeck, "Damage Identification on the Z24 bridge using vibration monitoring," Smart Materials and Structures, vol. 10, pp. 512-517, 2001. 